\title{
Analysis of a Simple Probe for In-Situ Resistivity Measurements
}

\author{
Jens Munk ${ }^{1}$, Todd Petersen', Matt Cullin², William Schnabel ${ }^{3}$ \\ ${ }^{1}$ Department of Electrical Engineering, University of Alaska Anchorage, Anchorage, AK, USA \\ ${ }^{2}$ Department of Mechanical Engineering, University of Alaska Anchorage, Anchorage, AK, USA \\ ${ }^{3}$ University of Alaska Fairbanks, Fairbanks, AK, USA \\ Email: jmunk2@alaska.edu
}

How to cite this paper: Munk, J., Petersen, T., Cullin, M. and Schnabel, W. (2017) Analysis of a Simple Probe for In-Situ Resistivity Measurements. Journal of Water Resource and Protection, 9, 1-10. http://dx.doi.org/10.4236/jwarp.2017.91001

Received: November 1, 2016

Accepted: January 6, 2017

Published: January 10, 2017

Copyright (c) 2017 by authors and Scientific Research Publishing Inc. This work is licensed under the Creative Commons Attribution International License (CC BY 4.0).

http://creativecommons.org/licenses/by/4.0/ (c) (i) Open Access

\begin{abstract}
We present a probe factor for a simple measurement device, which can be used to determine in-situ electrical resistivity in soils or other penetrable bodies. The probe is primarily sensitive to the material immediately surrounding it and therefore is ideal for determining localized conductivities. The geometry of the probe can be scaled to effectively adjust the region of interest. The calibration, or "probe factor" is a function of the geometry, as well as the electrode configuration. Results are presented assuming a Wenner array configuration, however they can easily be extended to other geometries, such as the Schlumberger or dipole-dipole array.
\end{abstract}

\section{Keywords}

Electrical Resistivity Measurements, Soil Moisture, Electrical Conductivity, Geophysical Measurement

\section{Introduction}

Measurements of a materials electrical resistivity can provide useful information for, indirectly determining soil moisture [1], assisting in the design of cathodic protection systems to prevent corrosion in buried metal structures [2], determining electrical substation grounding characteristics [3], measuring subsurface hydrological properties [4], and the extent of sub-seafloor sediment [5] [6] in oceanographic studies, to name but a few. Electrical Resistivity Tomography (ERT) also provides a useful geophysical tool in imaging resistivity variations in the subsurface [7].

This paper describes a simple probe to be used for in-situ resistivity measurements. We derive a probe factor based on the geometry of our device, which is given by an exact analytic solution of the boundary value problem. Our moti- 
vation for developing this probe was the need for an in-situ device to measure soil moisture over time within a free-draining lysimeter. Since the probe measures electrical resistivity directly, it is also necessary to relate this value to soil moisture through experimental measurements [1].

Analysis of this probe is similar to those used in bore-hole boundary value problems, specifically those employing an integral equation approach (see for example, Zhang [8]; Tsang [9]; Gianzero [10]). The current application is interested in material properties near the probe, and hence a more accurate representation of the probe current source(s) is required. The associated boundary value problem for the probe is solved in Appendix A, using an integral equation approach and assuming a homogeneous media where the conducting rings are placed over an insulated rod. The derivation in Appendix B includes the effect of a planar ground surface.

The mathematical formulation of our probe is based on a Wenner [11] style four point electrode configuration, however the results can easily be extended to other array geometries, such as a Schlumberger or a dipole-dipole [12]. For the Wenner method current is sourced through the outside points and the voltage is measured at the inside points, as shown Figure 1. The Wenner method has been adapted to very thin ring conductors around an insulating rod by Won [6]. In the case where the probe is to be driven into a soil mixture rather than a wet environment, it is necessary to expand the conducting rings to have finite thickness to ensure a good electrical connection of the probe and the surrounding material. The conducting bands having a thickness requires a new derivation for the probe factor to relate the apparent resistivity to actual resistivity of the material.

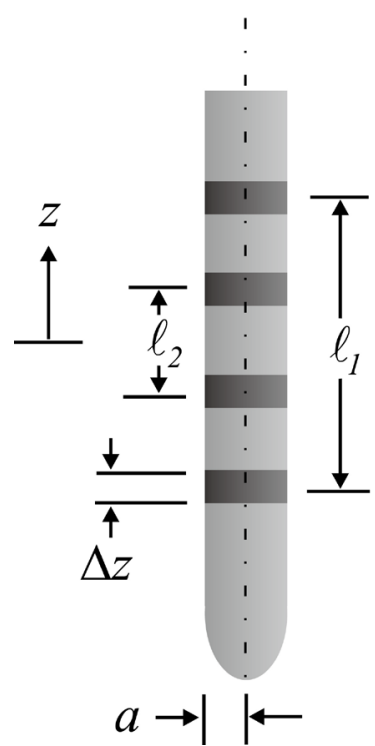

(a)

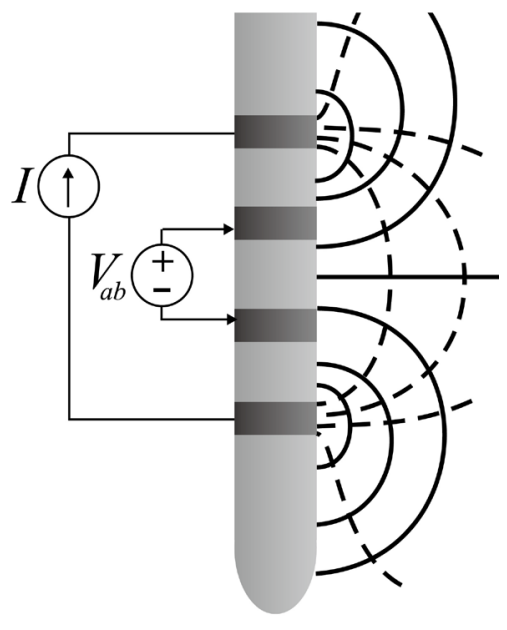

(b)

Figure 1. A sketch of the resistivity probe (a) with the associated equipotential lines shown as solid and the electric field/current density lines shown as dashes shown in (b). 
Experimental results are presented for comparison of the derived, simulated, and measured probe factor.

\section{Mathematical Formulation}

The probe, shown in Figure 1, consists of insulating rod of radius a, with four conducting rings of width $\Delta z$ with the outer and inner rings separated by a mean distance $\ell_{1}$ and $\ell_{2}$, respectively. Placing a known current across the two outer conducting rings and measuring the resulting potential across the two inner rings, the resistivity/conductivity of the surrounding material can be determined. This is achieved by first solving the associated boundary value problem, as derived in Appendix A.

In this derivation it is assumed that the electrical conductivity $\sigma$ is constant in the vicinity surrounding the probe so that in cylindrical coordinates $(\rho, \phi, z)$ the electric potential is given by,

$$
V(\rho, z)=\frac{-I}{\sigma \pi^{2} a \Delta z} \int_{0}^{+\infty} \frac{1}{k^{2}} \frac{K_{0}(k \rho)}{K_{1}(k a)} G(k) \sin k z \mathrm{~d} k
$$

where the probe is centered at $z=0, I$ is the measured current and

$$
G(k) \equiv \cos k \delta^{+}-\cos k \delta^{-},
$$

with $\delta^{+} \equiv\left(\ell_{1}+\Delta z\right) / 2, \quad \delta^{-} \equiv\left(\ell_{1}-\Delta z\right) / 2$, and $\delta_{2}^{-} \equiv\left(\ell_{2}\right) / 2$. Here the ground surface effect is neglected, however it is include later. Based on (1), a sketch of the potential as well as the current density $J$, given by

$$
J=-\sigma \nabla V \text {, }
$$

is shown in Figure 1. Notable in the figure is the localization of the fields near the probe electrodes.

The relationship between the apparent resistivity $\rho_{a}$, the applied current $I$ and measured voltage $V_{a b}$ is given by,

$$
\rho_{a}=\frac{R}{p f}
$$

where $R \equiv V_{a b} / I$, with $p f$ the "probe factor" defined as the quantity,

$$
p f \equiv \frac{2}{\pi^{2} a \Delta z} \int_{0}^{+\infty} \frac{1}{k^{2}} \frac{K_{0}(k a)}{K_{1}(k a)} G(k) \sin k \delta_{2}^{-} \mathrm{d} k .
$$

The integral given in (5) cannot be solved in closed form, and must therefore evaluated numerically. However, since the probe factor is solely a function of geometry, that is $p f=p f\left(\ell_{1}, \ell_{2}, a, \Delta z\right)$, it need only be calculated once for a given probe.

Figure 2 shows variations in the calculated probe factor for a probe radius $a$ and conductor thickness $\mathrm{dz}$ are independently varied from $0.5 \mathrm{~cm}$ to $2.0 \mathrm{~cm}$, with the electrode spacing $\ell=10.0 \mathrm{~cm}$ (a sensitivity analysis also indicated that the probe factor was generally less sensitive to variations in $\ell$ ).

With the ground interface included the expression for the probe factor is modified slightly and is given by,

$$
p f_{g}=\frac{4}{\pi^{2} a \Delta z} \int_{0}^{+\infty} \frac{1}{k^{2}} \frac{K_{0}(k a)}{K_{1}(k a)} G(k) \sin ^{2} k d \sin k \delta_{2}^{-} \mathrm{d} k,
$$


where $d$ is the depth to the center of the probe from the ground surface, and the subscript $g$ indicates that the air-earth interface is included. As expected the surface interface modifies $p f_{g}$ only slightly when the probe is very near the surface. This is indicated in Figure 3, which shows the calculated $p f_{g}$ as a function of probe depth $d$ for $a=1.0 \mathrm{~cm}, \Delta z=1.5 \mathrm{~cm}$ and $\ell=10.0 \mathrm{~cm}$. Noting the scale used for Figure 3, it is likely that the ground interface can be neglected in most applications.

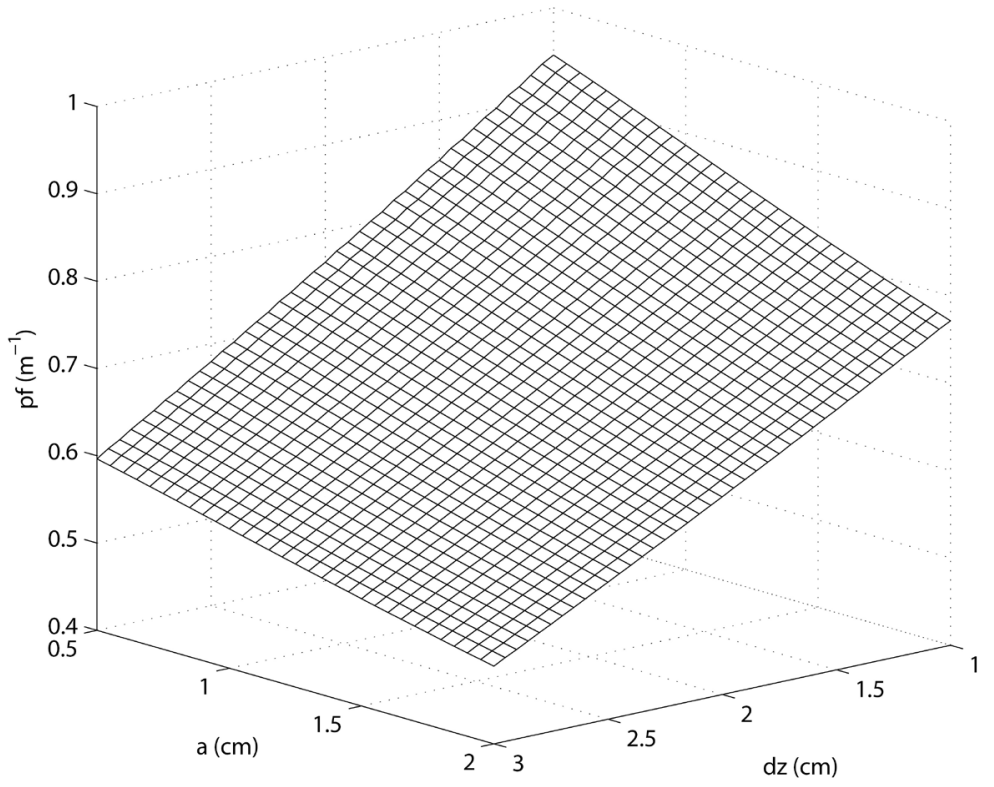

Figure 2. Resistivity probe factor, $p f$, as a function of probe radius $a$ and conductor thickness $\mathrm{d} z$, with $\ell_{1}=20.0 \mathrm{~cm}$, and $\ell_{2}=\ell_{1} / 3$.

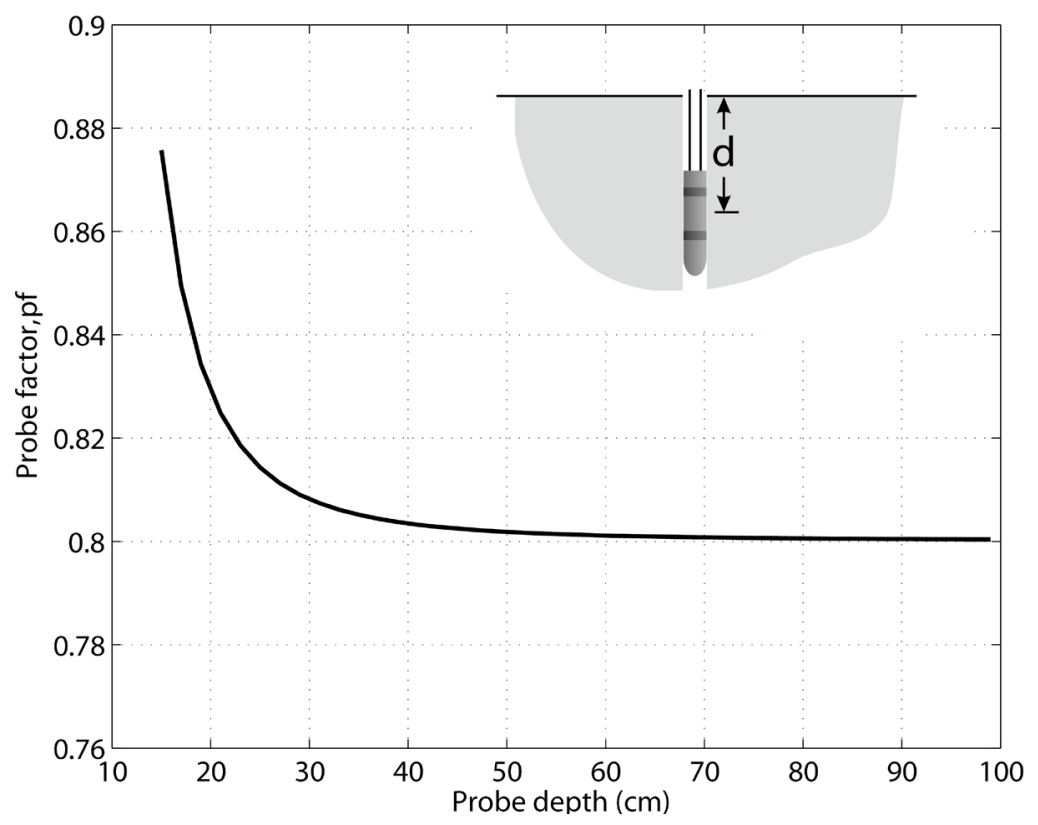

Figure 3. Resistivity probe factor, $p f$, as a function probe depth $d$ for $a=1.0 \mathrm{~cm}, \Delta z=1.5 \mathrm{~cm}$, and $\ell_{1}=20.0 \mathrm{~cm}$. 


\section{Results}

An experiment was conducted by submerging a custom built probe in a barrel of a salt water solution. Salt was incrementally added to the solution increasing its conductivity. The conductivity of the water was then measured and verified with a VWR Symphony conductivity probe (model number 11388 - 382). Once a voltage was applied to the outer rings of the probe, the electrical current through the outer rings and the voltage on the inner rings were measured with an Agilent Digital Multimeter (model number 34410a). The probe was submerged to approximately the same depth and readings were recorded for current and voltage. Next, the probe was then extracted, dried, and the process repeated for five measurements at each solution conductivity.

The probe constructed for experimental verification of (5) was designed and built in units of inches. Converted to centimeters they are; $a=0.9525 \mathrm{~cm}$, $\Delta z=1.27 \mathrm{~cm}, \ell_{1}=15.24 \mathrm{~cm}$, and $\ell_{2}=5.08 \mathrm{~cm}$. Surface to probe center depth was $21.59 \mathrm{~cm}$. Simulations of this probe were also conducted in COMSOL Multiphysics with a set material conductivity taken from one of the experimental measured levels.

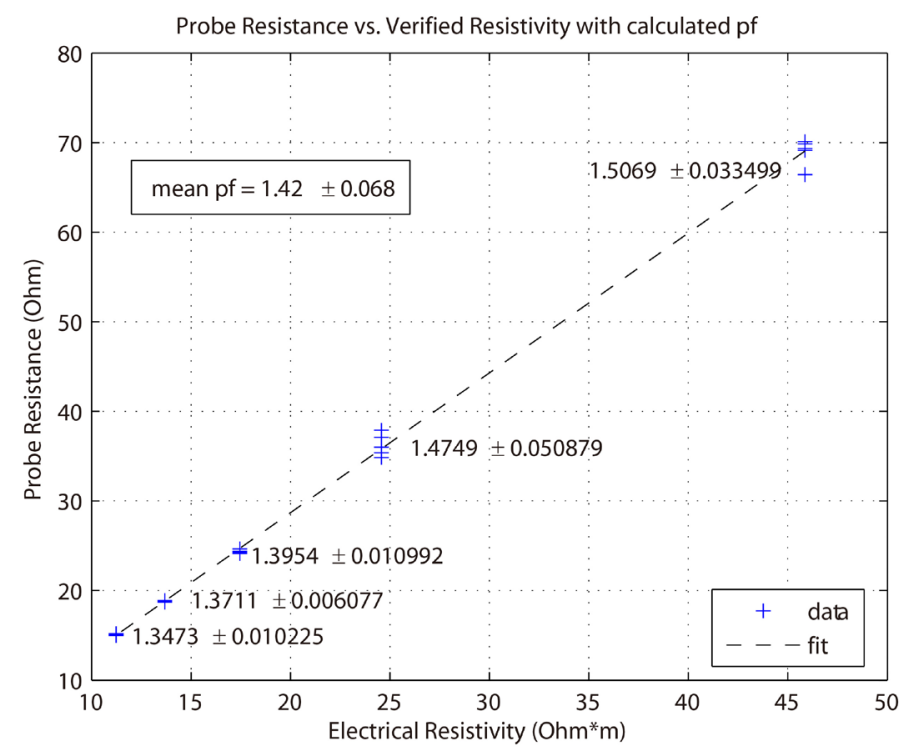

Figure 4. Graph of experimentally measured probe resistance versus verified fluid resistivity. Experimentally derived probe factor at each known resistivity for each set of measurements along with a linear curve fit and average probe factor are also presented.

Table 1. Analytical, numerical, and experimentally derived probe factors for the as-built probe

\begin{tabular}{cc}
\hline Method & Probe factor \\
\hline Analytical pf & $1.365 \mathrm{~m}^{-1}$ \\
Analytical w/ grounding $p f_{g}$ & $1.382 \mathrm{~m}^{-1}$ \\
COMSOL Multiphysics $p f$ & $1.379 \mathrm{~m}^{-1}$ \\
Experimental Meas. pf & $1.42 \pm 0.677 \mathrm{~m}^{-1}$ \\
\hline
\end{tabular}


Analytical, numerical, and experimentally derived probe factors for the as-built probe are presented in Table 1. The plot of the experimental measurements in Figure 4 shows that there is greater uncertainty in the measurements at higher resistances. The derived probe factor at each conductivity level is shown along with its uncertainty. The experimental pf differs from the computed value with the grounding effects by less than the standard deviation of the measurements. Simulations performed in COMSOL are also in agreement with the analytical and experimentally derived values for the probe factor.

\section{Conclusion}

We derive a mathematical expression for the probe-factor of a simple device for use in it-situ resistivity measurements. Our derivation is based on a Wenner array configuration, however our results are easily extended to include other geometries, such as the Schlumberger and the dipole-dipole arrays. Comparisons of our mathematically derived probe-factor with measured, and numerically derived results show excellent agreement.

\section{References}

[1] Schnabel, W.E., Munk, J., Abichou, T., Barnes, D., Lee, W. and Pape, B. (2012) Assessing the Performance of a Cold Region Evapotranspiration Landfill Cover Using Lysimetry and Electrical Resistivity. International Journal of Phytoremediation, 14, 61-75. https://doi.org/10.1080/15226514.2011.607870

[2] Jones, D.A. (1996) Principles and Prevention of Corrosion. 2nd Edition, Prentice Hall, Upper Saddle River.

[3] Baishiki, R.S., Osterberg, C.K. and Dawakibi, F. (1987) Earth Resistivity Measurements Using Cylindrical Electrodes at Short Spacings. IEEE Transactions on Power Delivery, PWRD-2, 64-71. https://doi.org/10.1109/TPWRD.1987.4308074

[4] Sherrod, L., Sauck, W. and Werkema Jr., D.D. (2011) A Low-Cost, In Situ Resistivity and Temperature Monitoring System. Ground Water Monitoring and Remediation, 32, 31-39. https://doi.org/10.1111/j.1745-6592.2011.01380.x

[5] Wheatcroft, R.A., Stevens, A.W. and Johnson, R.V. (2007) In Situ Time-Series Measurements of Subseafloor Sediment Properties. IEEE Journal of Oceanic Engineering, 32, 862-871. https://doi.org/10.1109/JOE.2007.907927

[6] Won, I.J. (1987) The Geometrical Factor of a Marine Resistivity Probe with Four Ring Electrodes. IEEE Journal of Oceanic Engineering, 12, 301-303. https://doi.org/10.1109/joe.1987.1145234

[7] Loke, M.H. and Barker, R.D. (1996) Rapid Least-Squares Inversion of Apparent Resistivity Pseudo-Sections Using Quasi-Newton Method. Geophysical Prospecting, 48, 131-152. https://doi.org/10.1111/j.1365-2478.1996.tb00142.x

[8] Zhang, G.J. and Shen, L.C. (1984) Response of a Normal Resistivity Tool in a Borehole Crossing a Bed Boundary. Geophysics, 49, 142-148. https://doi.org/10.1190/1.1441645

[9] Tsang, L., Chan, A.K. and Gianzero, S. (1984) Solution of the Fundamental Problem in Resistivity Logging with a Hybrid Method. Geophysics, 49, 1596-1604. https://doi.org/10.1190/1.1441568

[10] Gianzero, S. and Anderson, B. (1982) An Integral Transform Solution to the Fundamental Problem in Resistivity Logging. Geophysics, 47, 946-956. 
https://doi.org/10.1190/1.1441362

[11] Wenner, F. (1915/16) A Method of Measuring Resistivity. National Bureau of Standards, Scientific Bulletin, 12, 478-496.

[12] Telford, W.M., Geldart, L.P. and Sheriff, R.E. (1990) Resistivity Methods. In: Applied Geophysics, 2nd Edition, Cambridge Univ. Press, Cambridge, UK, 353-358. https://doi.org/10.1017/cbo9781139167932.012

[13] Koefoed, O. (1968) The Application of the Kernel Function in Interpreting Geoelectrical Resistivity Measurements. Series 1-No. 2. Gebrüder Borntraeger, Berlin.

[14] Watson, G.N. (1962) A Treatise on the Theory of Bessel Functions. 2nd Edition, Cambridge Univ. Press, Cambridge, UK. 


\section{Appendix}

\section{A. Uniform Ground}

Beginning with the equation of continuity for the current density $J$, which for the static case is given by,

$$
\nabla \cdot J=0 .
$$

The current density at a point is related to the electric field through $J=\sigma E$, where $\sigma$ is the electrical conductivity and $E=-\nabla V$, with $V$ the static electric potential.

Since the probe is sensitive only to the local region surrounding it we assume that $\sigma$ is approximately constant so that the potential satisfies Laplace's equation, namely

$$
\sigma \nabla^{2} V=0
$$

Employing a cylindrical coordinate system, specified by $(\rho, \phi, z)$, with the length of the probe is along the $z$-axis it is clear that our solution must be independent of $\phi$. Then the Laplacian reduces to,

$$
\nabla^{2} V=\frac{1}{\rho} \frac{\partial}{\partial \rho}\left(\rho \frac{\partial V}{\partial \rho}\right)+\frac{\partial^{2} V}{\partial z^{2}}=0
$$

with $V=V(\rho, z)$. Assuming a solution of the form, $V(\rho, z)=R(\rho) Z(z)$.

Subject to the boundary condition that the potential vanish at $\rho \rightarrow \infty$ the general solution for the potential is then given by,

$$
V(\rho, z)=\int_{-\infty}^{+\infty} F(k) K_{0}(k \rho) \mathrm{e}^{-j k z} \mathrm{~d} k
$$

where $F(k)$ (referred to as the "kernel function" [13]) is an unknown function of $k$ to be determined from the boundary conditions. Equation (10) is a Fourier integral wherein by the inverse Fourier transformation $F(k)$ can be determined via,

$$
F(k) K_{0}(k \rho)=\frac{1}{2 \pi} \int_{-\infty}^{+\infty} V(\rho, z) \mathrm{e}^{j k z} \mathrm{~d} z .
$$

Next boundary conditions are established on the surface of the probe, where it is assumed that the probe is insulated, except for the conducting bands, which are also assumed to be ideal conductors. Thus the surface of the probe represent a no--flow boundary, except at the two conducting bands where the current density has only a radially directed component. Since the current density is given by

$$
J(\rho, z)=-\sigma\left(\frac{\partial V}{\partial \rho} \hat{\rho}+\frac{\partial V}{\partial z} \hat{z}\right)
$$

We obtain for the radially directed component at the surface of the probe $(\rho=a)$,

$$
J(a, z)=\sigma \int_{-\infty}^{+\infty} F(k) k K_{1}(k a) \mathrm{e}^{-j k z} \mathrm{~d} k
$$

where we have used that Watson [14]

$$
\frac{\partial}{\partial \rho} K_{0}(k \rho)=-k K_{1}(k \rho) .
$$


Since the conducting rings constitute equipotential boundaries the current densities must also be constant on along their surface. We can therefore express the current density at the surface of the probe as,

$$
J_{\rho}(a, z)=\frac{I}{\Delta z 2 \pi a}\left\{\left[u\left(z_{2}\right)-u\left(z_{1}\right)\right]-\left[u\left(z_{4}\right)-u\left(z_{3}\right)\right]\right\}
$$

where

$$
z_{1}=z-\delta^{+}, \quad z_{2}=z-\delta^{-}, \quad z_{3}=z+\delta^{-}, \quad z_{4}=z+\delta^{+},
$$

$I$ is the measured current, $\ell_{1}$ is the mean separation between the two outer conducting rings, $\Delta z$ the ring width, as shown in Figure 1(a), with

$$
\delta^{+} \equiv\left(\ell_{1}+\Delta z\right) / 2, \quad \delta^{-} \equiv\left(\ell_{1}-\Delta z\right) / 2 .
$$

Equation (13) is solved making use of the inverse Fourier transform. This gives,

$$
\begin{aligned}
\sigma F(k) k K_{1}(k a) & =\frac{1}{2 \pi} \int_{-\infty}^{+\infty} J_{\rho}(a, z) \mathrm{e}^{j k z} \mathrm{~d} z \\
& =\frac{I}{2 \pi^{2} a \Delta z} \frac{1}{j k} G(k)
\end{aligned}
$$

where

$$
G(k) \equiv \cos k \delta^{+}-\cos k \delta^{-}
$$

Solving for $F(k)$ in equation yields,

$$
F(k)=\frac{-j I}{\sigma 2 \pi^{2} a \Delta z} \frac{1}{k^{2}} \frac{1}{K_{1}(k a)} G(k)
$$

so that the electrical potential can be expressed as,

$$
V(\rho, z)=\frac{-I}{\sigma \pi^{2} a \Delta z} \int_{0}^{+\infty} \frac{1}{k^{2}} \frac{K_{0}(k \rho)}{K_{1}(k a)} G(k) \sin (k z) \mathrm{d} k
$$

given that the term

$$
\frac{1}{k^{2}} \frac{K_{0}(k \rho)}{K_{1}(k a)} G(k)
$$

within the integrand is odd with respect to the variable of integration $k$. Hence only the term $-j \sin k z$ associated with $\mathrm{e}^{-j k z}$ contributes to the integral when evaluated over our limits of integration. The expression for $V$ given in (18) is preferable since it does not contain any imaginary terms, indicating that the potential is purely real, as it must be.

The final task is to relate the measured potential $V_{a b}$ to the known current, $I$ applied across the two outer conducting rings. First define the term,

$$
\delta_{2}^{-}=\left(\ell_{2}\right) / 2
$$

then by definition,

$$
\begin{gathered}
V_{a b}=V\left(a, \delta_{2}^{-}\right)-V\left(b,-\delta_{2}^{-}\right) \\
=\frac{2 I}{\sigma \pi^{2} a \Delta z} \int_{0}^{+\infty} \frac{1}{k^{2}} \frac{K_{0}(k a)}{K_{1}(k a)} G(k) \sin \left(k \delta_{2}^{-}\right) \mathrm{d} k .
\end{gathered}
$$

Hence, the ratio between the measured voltage and applied current $V_{a b} / I$ is given by, 


$$
\frac{V_{a b}}{I}=\frac{2}{\sigma \pi^{2} a \Delta z} \int_{0}^{+\infty} \frac{1}{k^{2}} \frac{K_{0}(k a)}{K_{1}(k a)} G(k) \sin \left(k \delta_{2}^{-}\right) \mathrm{d} k
$$

Define $R \equiv V_{a b} / I$, then the apparent resistivity $\rho_{a}$ is given by

$$
\rho_{a}=\frac{1}{\sigma}=\frac{R}{p f},
$$

with the probe factor given by,

$$
p f=\frac{2}{\pi^{2} a \Delta z} \int_{0}^{+\infty} \frac{1}{k^{2}} \frac{K_{0}(k a)}{K_{1}(k a)} G(k) \sin \left(k \delta_{2}^{-}\right) \mathrm{d} k
$$

\section{B. Uniform Half-Space}

To include the effect of the ground surface, image theory is used to enforce the no-flow boundary at the ground-air interface. Assuming the probe is buried a depth $d$ as measured from the center of the probe to the ground surface,and assuming the $z$-axis to be centered at the probe (as previously), then

$$
F_{g}(k)=F(k)\left(1-\mathrm{e}^{-j 2 k d}\right)
$$

with $F_{g}$ the modified kernel function with the ground effect included. In obtaining (25) the method of images was used as was the shifting property of the Fourier transform. Then, the potential in the lower half-space is given by,

$$
V(\rho, z)=\int_{-\infty}^{+\infty} F_{g}(k) K_{0}(k \rho) \mathrm{e}^{-j k z} \mathrm{~d} k
$$

where again the even/odd symmetry in our integrand was used to simplify the resulting integral. Following the same general procedure as in the previous case gives the modified probe factor,

$$
p f_{g}=\frac{4}{\pi^{2} a \Delta z} \int_{0}^{+\infty} \frac{1}{k^{2}} \frac{K_{0}(k a)}{K_{1}(k a)} G(k) \sin ^{2}(k d) \sin \left(k \delta_{2}^{-}\right) \mathrm{d} k,
$$

with the subscript $g$ indicating the ground effect.

\section{Submit or recommend next manuscript to SCIRP and we will provide best} service for you:

Accepting pre-submission inquiries through Email, Facebook, LinkedIn, Twitter, etc. A wide selection of journals (inclusive of 9 subjects, more than 200 journals)

Providing 24-hour high-quality service

User-friendly online submission system

Fair and swift peer-review system

Efficient typesetting and proofreading procedure

Display of the result of downloads and visits, as well as the number of cited articles

Maximum dissemination of your research work

Submit your manuscript at: http://papersubmission.scirp.org/

Or contact jwarp@scirp.org 